canxi máu. Mức độ đau sau mổ được chia thành các mức: đau nhẹ, đau vừa và đau nặng. Kết quả nghiên cứu của chúng tôi trong 24 giờ sau mổ mức độ đau của bệnh nhân gặp nhiểu nhất là đau nhẹ chiếm $62,2 \%$ đối với mổ mở và $82,2 \%$ đối với phẫu thuật nội soi $(p<0,05)$.Trong nghiên cứu của Trịnh Minh Tranh (2013) nhóm phẫu thuật mở có tỷ lệ đau vừa là $62,3 \%$ và đau nhe là 14,2\%; nhóm PTNS có tỉ lệ đau nhe là 85,6\%[3]. Trong nghiên cứu của Lê Văn Giáp (2014) về phẫu thuật mở thì tỷ lệ đau nhe là $10 \%$ và đau vừa là $90 \%$ [4]. Có thể thây PTNS đau sau mổ ít hơn so với phẫu thuật mở. Tỷ lệ rất hài lòng về kết quả điều trị ở nhóm PTNS $(80 \%)$ cao hơn ở nhóm phẫu thuật mở $(75,6 \%)$, $(p<0,05)$. Trong nghiên cứu của Trịnh Minh Tranh (2013) có 94,6 \% bệnh nhân hài lòng đối với nhóm PTNS, tỷ lệ này ở nhóm phẫu thuật mở là 76,4\%[3]. Trong nghiên cứu về PTNS tuyến giáp của Akira (2008) có $96,4 \%$ bệnh nhân hài lòng về kết quả điều trị[8]. Có thể thấy bệnh nhân ở nhóm PTNS hài lòng hơn về kết quả điều trị so với nhóm phẫu thuật mở, đặc biệt là vấn đề thẩm mỹ.

\section{KẾT LUÂ̂N}

Phẫu thuật mở và PTNS đường nách vú đều có thể áp dụng trong điều trị u tuyến giáp lành tính với tính hiệu quả và độ an toàn cao, tỉ lệ tai biến trong mổ và biến chứng sau mổ thấp. Trong đó PTNS đường nách vú đảm bảo yêu cầu về thẩm mĩ ngày càng cao của bệnh nhân đồng thời mức độ đau sau mổ ít hơn so với phẫu thuật mở.

\section{TÀI LIÊUU THAM KHẢO}

1. Tunbridge W.M., Evered D.C., Hall R., et al. (1977). The spectrum of thyroid disease in a community: the Whickham survey. Clin Endocrinol (Oxf), 7(6), 481-493.

2. Gharib H, Papini E, Garber JR, et al (2016). American Association of Clinical Endocrinologists, American College of Endocrinology, and Associazione Medici Endocrinologi 'Medical Guidelines for Clinical Practice for the Diagnosis and Management of Thyroid Nodules - 2016 Update Appendix. Endocrine Practice, 22,1-60.

3. Trinh Minh Tranh (2013), Nghiên cứu chỉ định điểu trị bướu giáp đơn nhân bằng phẫu thuâtt nôi soi, Luận án tiến sỹ Y học, Đại học Y dược tp Hồ Ċhí Minh.

4. Lế Văn Giáp (2014), Nghiên cứu đăc điểm lâm sàng, cận lâm sàng và đánh giá kểt quả phẫu thuật u lành tính một thùy tuyên giáp qua đường cổ bên, Luân văn Thạ́ sĩ Y học, Đai học Y Hà Nôi, HN.

5. Hershman J. M. (2005). Perchlorate and thyroid function: what are the environmental issues?. Thyroid: official journal of the American Thyroid Association, 15(5), 427-431.

6. Đinh Xuân Cường (2004). "Nghiên cứu đặc điểm lâm sàng, mô bệnh học và kết quả điều trị phẩu thuật ung thư tuyến giáp tại bệnh viện $\mathrm{K}^{\prime \prime}$. Luân văn thạc sĩ y hoc, Hà Nội, 33 - 48.

7. Trần Ngọc Lương (2011). Đ̇ánh giá kết quả phẫu thuật nội soi điêu trị bướu giáp lành tính. Tạp ch phẩu thuật nội soi và nội soi Việt Nam. 1(2), 20-24.

8. Sasaki A, Nakajima J, Ikeda K, Otsuka K, Koeda K, Wakabayashi G (2008). Endoscopic Thyroidectomy by the Breast Approach: A Single Institution's 9-year Experience. World journal of surgery, 232,381-385.

\title{
HOÀN THIỆN QUY TRÌNH XA TRI LẬP THỂ TRÊN HỆ PHANTOM SỬ DỰG MÁY GIA TỐC VERSA-HD TẠI BỆNH VIỆN K
}

\section{TÓM TẮT}

Mục tiêu: Xa trị lập thể trên nền tảng máy gia tốc tuyến tính là một lựa chọn tối ưu cho những bệnh nhân có chỉ định điều trị. Mỗi cơ sở được trang bị hệ thống máy và thiết bị phụ trợ khác nhau. Nghiên cứu triển khai thực hiện xạ trị lập thể trên hệ phanton hình đâu người để bưởc đẩu chuẩn hóa quy trình xạ trị lập thể trên nền tảng máy gia tốc tuyến tính VersaHD tại bệnh viện $K$. Đối tượng và phương pháp nghiên cứu: Nghiên cứu thực hiện dựa trên các hướng dẫn

\section{${ }^{1}$ Bệnh viện $K$}

Chịu trách nhiệm chính: Lê Văn Tình

Email: tinhbvk@gmail.com

Ngày nhận bài: 4.8.2021

Ngày phản biên khoa họ: 1.10 .2021

Ngày duyệt bài: 8.10.2021
Lê Văn Tình ${ }^{1}$, Nguyễn Thị Thơm ${ }^{1}$, Nguyễn Thanh Bình ${ }^{1}$

quốc tế của ICRU và AAPM về quy trình lập kế hoạch và đánh giá kế hoạch. Ngoài ra, nghiên cứu thực hiện quy trình xạ trị lập thể trên phantom giả lập đầu người với kích thước và vị trí u khác nhau. Kết quả: Đưa ra quy trình lâp kế hoach và đánh giá kế hoach xa tri tai cơ sở, đánh giá được khả năng thực thi của cắc thiết bị hiện có tai cơ sở trong việc thực hành xa phấu. Kết luận: Nghiển cứu khẳng định khả năng thực hiện kĩ thuật xạ phẫu trên nền tảng máy gia tốc và các thiết bị đi kèm của cơ sở trước khi thực hiện điêuu trị trên bệnh nhân.

\author{
SUMMARY \\ STEREOTACTIC RADIATION THERAPY \\ PROCESS ON PHANTOM SYSTEM USING \\ VERSA-HD LINEAR ACCELERATOR AT \\ VIETNAM NATIONAL CANCER HOSPITAL
}


Objective: Stereotactic radiation therapy (SRT) based on linear accelerators is an optimal choice for patients with indications. Each facility is equipped with different machine systems and ancillary equipment. To study and implement SRT on head phanton system to initially standardize the SRT procedure on the basis of the VersaHD linear accelerator at K hospital. Subject and Method: A practical study currently based on ICRU and AAPM guidelines on the planning and evaluation process. In addition, the study performed SRT procedures on head phantom with different tumor sizes and locations. Result: Provide a process for planning and evaluating radiotherapy plans at the facility, evaluating the performance of existing equipment at the facility in practicing SRT. Conclusion: The study confirmed the facility's ability to perform SRT on the basis of accelerators and associated equipment before performing treatment on patients.

\section{I. ĐĂT VẤN ĐỀ}

Trong nhiều thập kỷ qua, khoa học kĩ thuật luôn có một vai trò quan trọng trong sự phát triển của xạ trị. Đỉnh cao của sự phát triển công nghệ trong xạ trị là xạ phẫu SRS/SBRT. Xạ phẫu với nhiều ưu việt là không xâm lấn, bệnh nhân không cần lưu trú dài ngày tại bệnh viện, giảm thiểu tối đa liều xạ vào mô lành hoặc thậm chí giữ được nguyên vẹn các cơ quan liền kề sau điều trị. Các máy xạ phẫu chuyên biệt như Gamma Knife, Cyber Knife...ra đời là cánh tay phải của người "làm phẫu thuật" bằng xạ trị, nhằm loại bỏ được khối u nhưng vẫn bảo vệ được các cơ quan nguy cấp cho bênh nhân. Ngày nay, xạ phẫu trên hệ thống máy gia tốc cũng là một lựa chọn, khi thực hiện kĩ thuật đòi hỏi độ chính xác cao trong việc phân phổi liều điều trị tới bệnh nhân bởi liều xạ lớn trong một phân liều. Bên cạnh đó, việc sử dụng nhiều dụng cụ cố định để hố trợ cố định bệnh nhân trong khi sứ dùng các chùm tia không đồng phẳng để đạt được sự phân bố liều tối ưu (Non - coplanar) có thể dấn đến các va chạm nguy hiểm tới bệnh nhân và thiết bị. Cùng với đó là các quy trình kỹ thuất trong khám và điều trị ung bướu do Bộ Y tế ban hành vẫn chưa có quy trình xạ trị lập thể trên nền tảng máy gia tốc tuyến tính. Chính vì những điều này, nhóm nghiên cứu lựa chọn đề tài nghiên cứu này nhằm đánh giá tính khả thi của kỹ thuật xạ phẫu trên nền tảng máy gia tốc và từ đó xây dựng ra các quy trình kỹ thuật chuẩn, phù hợp với trang thiết bị của cơ sở được trang bị để phục vụ công tác chuyên môn lâm sàng.

\section{II. ĐỐI TƯợNG VÀ PHƯƠNG PHÁP NGHIÊN CứU 2.1. Đối tượng và thiết bị nghiên cứu Đối tượng nghiên cứu:}

- Nghiên cứu các quy trình lập kế hoạch (planning) và đánh giá kế hoạch (evaluation) cho kỹ thuật xạ trị lập thể.

- Nghiên cứu các quy trình kiểm tra trước điều trị (pre-treatment verification) cho kỹ thuật xạ trị lập thể.

- Nghiên cứu thực hiện 10 kế hoạch trên phantom đầu người với các thể tích u và vị trí khác nhau. Khối u có đường kính dưới $10 \mathrm{~cm}^{3}$.

Thiết bị nghiên cứu:

- Nghiên cứu được thực hiện trên máy gia tốc Elekta (Stockholm, Thụy Điển) với hệ MLC agility (máy Infinity, Versa HD) với 160 cặp lá MLC dày $5 \mathrm{~mm}$, sử dụng mức năng lượng 6FFF MV (flat filter free) và 10FFF MV. Hệ thống lập kễ hoạch Monaco v5.11 có khả năng thực hiện kĩ thuật DCAT, VMAT hay dùng trong xạ trị lập thể.

- Giường điều trị Hexapod là hệ thống giường tự động với 6 bậc tự do (6-DOF), hệ thống chụp cone beam $\mathrm{CT}$.

- Thiết bị phantom đầu người.

- Thiết bị QA kế hoạch Dolphin với phần mềm COMPASS và thiết bị Arccheck được sử dụng trong nghiên cứu để kiểm chuẩn kế hoạch trước điêu trị. Dolphin gồm 1514 cảm biến (sensors) trong đó có 1513 buồng ion hóa phằng song song và 1 diode được đặt ở cạnh, được sắp xếp thành mang lưới. thể tích nhạy của các buồng ion hóa là $0,016 \mathrm{cc}$. Vùng hoạt động đo đạc của detector là $24 \mathrm{~cm} \times 24 \mathrm{~cm}$. Độ phân giải không gian là $5 \mathrm{~mm}$ với trường chiếu $14 \mathrm{~cm} \times 14 \mathrm{~cm}$ và $10 \mathrm{~mm}$ trong vùng ngoài trường chiếu $14 \mathrm{~cm} \times 14 \mathrm{~cm}$.

- ArCCHECK (ArCCHECK, Sun Nuclear, Melbourne, Hoa Kỳ). ArcCHECK gồm 1386 đâu dò diode với độ phân giải không gian $10 \mathrm{~mm}$ (vùng hoạt động của 1 detector là 0,8 x 0,8mm2).

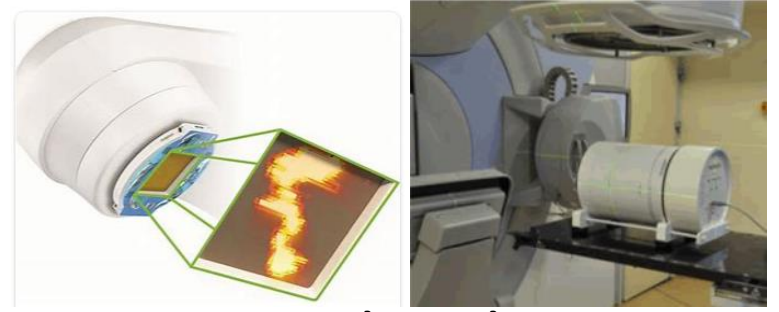

Hình 1: Thiêt bị kiểm chuẩn trước điều trị Dolphin (bên trái), Arccheck (bên phải).

\subsection{Phương pháp nghiên cứu}

\subsubsection{Quy trình nghiên cứu}

- Nghiên cứu áp dụng các quy trình của ICRU 91 và AAPM 101 cho máy gia tốc, các nghiên cứu và hướng dẫn đa trung tâm hay của từng trung tâm.

- Sử dụng thiết bi 2D - detector, kiểm tra 3D và đo liều điểm để kiểm tra các kế hoạch điều trị. 
Quy trình thực hiện một kế hoạch xạ trị lập thể vùng đầu cổ:

- Hình ảnh và mô phỏng: hình ảnh mô phỏng sử dụng trong lập kế hoạch xạ trị là ảnh CT mô phỏng, ngoài ra còn kết hợp với các hình ảnh MRI nhằm xác định chính xác các tổn thương cũng như tổ chức nguy cấp trong não.

- Xác định thể tích bia, tổ chức nguy cấp và lập kế hoạch điều trị: Bác sĩ xạ trị xác định các thể tích cần quan tâm trên hình ảnh MRI và $C T$. Kỹ thuật DCAT, VMAT được sử dụng trong lập kễ hoạch xạ trị với 1 hay nhiêu tâm điêu trị. Trong lập kế hoạch xạ trị lập thể việc thực hiện các trường chiếu không đồng phẳng là yếu tổ quan trọng nhằm tạo nhiêu hướng chiếu khác nhau, tạo ra độ dốc liều lớn. Kỹ thuật không đồng phẳng được thực hiện bằng cách quay giường điều trị, thêm vào đó là sự thay đổi góc quay của collimator để tạo hình dáng phù hợp của khối u cũng như giảm các cầu liêu trong kế hoạch nhiều thể tích bia. Trong kế hoạch xạ trị lập thể sử dụng chùm tia FFF với suất liều cao giúp giảm thời gian điều trị và độ dốc về liều lượng. Đặc biệt khi sử dụng đơn phân liều bởi sử dụng liểu lớn. Trong lập kế hoạch xạ trị lập thể, kích thước ô lưới là một vấn đề cần quan tâm, nhiều nghiên cứu chỉ ra các kích thước ô lưới tính toán dưới 3mm được lựa chọn trong tính toán, giá trị tối ưu thường sử dụng là $2 \mathrm{~mm}$.

- Đánh giá kế hoạch xạ trị: kế hoạch xạ trị lập thể được đánh giá theo ICRU91 và AAPM TG101

Đánh giá thể tích bia: ICRU91 chỉ ra với SRS/SRT cho trường trợp 1 tổn thương di căn não thì gần $100 \%$ PTV nên đạt liều chỉ định, ngoài ra cần đánh giá các thông số: $\mathrm{D} 50 \%$, Dmean, Dmean-min, Dmean-max, OAR, PRV, CI, GI.

Trong đó: $\mathrm{CI}=\frac{P T V \times P I V}{P T V_{P I V}^{2}} ; \mathrm{GI}=\frac{P I V_{\text {half }}}{P I V} ; \mathrm{PIV}$ : thể tích đường đồng liều chỉ định; $P I V_{\text {half }}$ : thể tích một nửa liều chỉ định.

Với thể tích nhỏ dưới $2 \mathrm{~cm}^{3}$, đánh giá $D_{\text {mean- }}$ $\min =D_{V-35 m^{3}}$ và $D_{\text {mean-max }}=D_{35 m^{3}}$

\subsection{2 Đánh giá tổ chức nguy cấp (TG101).}

- Kiểm chuấn kế hoạch trước điêu trị: được thực hiện theo AAPM TG218, đánh giá được thực hiện dựa trên tiêu chí gamma. Trong nghiên cứu này thực hiện kiểm định kế hoạch trên thiết bị Arccheck và Dolphin.

- Điều trị bệnh nhân: bệnh nhân được thực hiện chụp cone beam CT trước mỗi buổi điều trị, kết hợp với hệ thống bàn tự động Hexapod nhằm hạn chế sai số set up tối đa trước mỗi buổi điều tri.
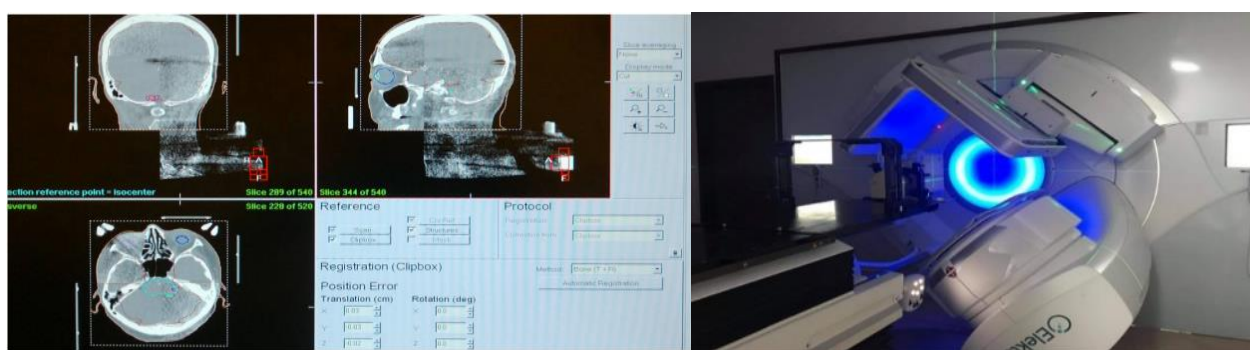

Hình 2. Chụp CBCT trước điều trị (bên trái), thực hiện điều trị (bên phải).

\section{KẾT QUẢ NGHIÊN CỨU \\ 3.1 Quy trình lập kế hoạch}

- Ảnh MRI lát cắt dày $1 \mathrm{~mm}$ hoăc tối thiểu $<2 \mathrm{~mm}$ trên máy CHT $1 \mathrm{~T}-3 \mathrm{~T}, \mathrm{CT}$ mố phỏng với độ dày lát cắt $1 \mathrm{~mm}$. Cả quy trình lập kế hoạch xạ trị kéo dài tối đa 2 tuần (nếu có thể thì thực hiện trong một tuần để nhằm tránh sự phát triển của khối u, đặc biệt các khối u ác tính.

- Import vào hê thống lập kế hoach Monaco, chồng chập các ảnh và vẽ các thể tích u và tổ chức nguy cấp. Lập kế hoạch xạ trị với các kĩ thuật DCAT hoặc VMAT, kích thước ô lưới tính toán $<3 \mathrm{~mm}$.

- Tối ưu hóa cung, tối ưu hóa kế hoạch.
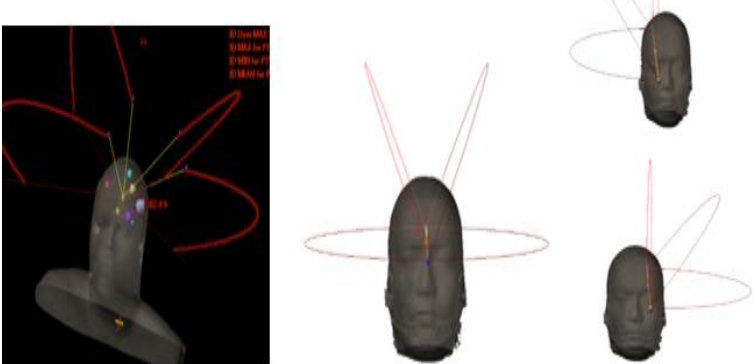

Hình 3: Một số thiêt kế cung, góc trong lập kế hoạch xạ phẫu [5],[7].

\subsection{Quy trình đánh giá kế hoạch}

- Đánh giá tổ chức nguy cấp theo TG101 [4] 


\begin{tabular}{|c|c|c|c|}
\hline & $1 \mathrm{Fx}$ & $3 \mathrm{Fx}$ & $5 \mathrm{Fx}$ \\
\hline Brain & $\mathrm{V}_{12,0 \mathrm{~Gy}} \leq 10,0 \mathrm{CC}$ & & \\
\hline Lens (Thüy tino lành) & $\mathrm{D}_{\max }<10,0 \mathrm{~Gy}$ & $\mathrm{D}_{\max }<10,0 \mathrm{~Gy}$ & $\mathrm{D}_{\max }<10,0 \mathrm{~Gy}$ \\
\hline Brainstem & $\mathrm{D}_{\max }<15,0 \mathrm{~Gy}$ & $\mathrm{D}_{\max }<23,1 \mathrm{~Gy}$ & $\mathrm{D}_{\max }<31,0 \mathrm{~Gy}$ \\
(Thân não) & $\mathrm{V}_{10,0 \mathrm{~Gy}} \leq 0,50 \mathrm{cC}$ & $\mathrm{V}_{18,0 \mathrm{~Gy}} \leq 0,5 \mathrm{cC}$ & $\mathrm{V}_{23,0 \mathrm{~Gy}} \leq 0,5 \mathrm{cC}$ \\
\hline Chiasm (Giao thoa thị) & $\mathrm{D}_{\max }<10,0 \mathrm{~Gy}$ & $\mathrm{D}_{\max }<17,4 \mathrm{~Gy}$ & $\mathrm{D}_{\max }<25,0 \mathrm{~Gy}$ \\
\hline Eye Ball (Bóng mắt) & $\mathrm{D}_{\max }<10,0 \mathrm{~Gy}$ & $\mathrm{D}_{\max }<17,4 \mathrm{~Gy}$ & $\mathrm{D}_{\max }<25,0 \mathrm{~Gy}$ \\
\hline Optic nerves & $\mathrm{D}_{\max }<10,0 \mathrm{~Gy}$ & $\mathrm{D}_{\max }<17,4 \mathrm{~Gy}$ & $\mathrm{D}_{\max }<25,0 \mathrm{~Gy}$ \\
(Thần kinh thị giác) & $\mathrm{V}_{8,0 \mathrm{~Gy}} \leq 0,20 \mathrm{cC}$ & $\mathrm{V}_{15,3 \mathrm{~Gy}} \leq 0,2 \mathrm{cC}$ & $\mathrm{V}_{23,0 \mathrm{~Gy}} \leq 0,2 \mathrm{cC}$ \\
\hline Spinal Cord & $\mathrm{D}_{\max }<14,0 \mathrm{~Gy}$ & $\mathrm{D}_{\max }<21,9 \mathrm{~Gy}$ & $\mathrm{D}_{\max }<30,0 \mathrm{~Gy}$ \\
(Tủy sống) & $\mathrm{V}_{10,0 \mathrm{~Gy}} \leq 0,35 \mathrm{cC}$ & $\mathrm{V}_{18 \mathrm{~Gy}} \leq 0,35 \mathrm{cC}$ & $\mathrm{V}_{23 \mathrm{~Gy}} \leq 0,35 \mathrm{cC}$ \\
& $\mathrm{V}_{7,0 \mathrm{~Gy}} \leq 1,20 \mathrm{cC}$ & $\mathrm{V}_{12,3 \mathrm{~Gy}} \leq 1,2 \mathrm{cC}$ & $\mathrm{V}_{14,5 \mathrm{~Gy}} \leq 1,2 \mathrm{cC}$ \\
\hline
\end{tabular}

3.3 Quy trình kiểm định kế hoạch trước điều trị. Kế hoạch sau khi được chấp nhận được thực hiện kiểm định 2D, 3D trên thiết bị Arccheck và Dolphin. Trong đó, chỉ số gamma $<1$ với tî lệ đạt trên $90 \%$ với tiêu chí $3 \% / 2 \mathrm{~mm}$ khi dùng Arccheck và $2 \% / 2 \mathrm{~mm} ; 2 \% / 1 \mathrm{~mm}$ với Dolphin.

\section{4 Ứng dụng trên phantom}

- Nghiên cứu tiến hành lập kế hoạch trên phantom: Đánh giá kế hoạch

$\mathrm{CI}_{\text {Paddick }}=0,87 \pm 0,12$

GIPaddick $=3,87 \pm 1,24$

- Kết quả kiểm định kế hoạch trên Dolphin với tiêu chí $(2 \% / 2 \mathrm{~mm})$ và $(2 \% / 1 \mathrm{~mm})$ có tỉ lệ gamma đạt là $96,1 \pm 3,5(\%)$ và $92,4 \pm 2,34(\%)$.

Trên thiết bị Arccheck với tiêu chí $(3 \% / 2 \mathrm{~mm})$ có tỉ lệ gamma đạt là $91,3 \pm 1,32$ (\%).

- Hệ thống giường điều trị Hexapod với sai số trục tịnh tiến $0,3-0,5 \mathrm{~mm}$ và trục quay là $0,3^{0}$. Nghiên cứu của Roper và cộng sự (2015) [4] chỉ ra sai số trục quay $0,5^{\circ}$ thì thể tích bia vẫn $\geq 95 \%$.

\section{BÀN LUÂNN}

Trong nghiên cứu này, chúng tôi đã tiến hành nghiên cứu các tài liệu và các công bố của tổ chức lớn như ICRU, AAPM nhằm đưa ra hoàn thiên các quy trình lập kế hoạch xạ trị và đánh giá kế hoạch trước điều trị (AAPM TG101，218; ICRU91). Một số điểm chú ý trong xạ trị lập thể não đặc biệt là khối u di căn là cả quy trình lập kế hoạch xạ trị kéo dài tối đa 2 tuân (nếu có thể thì thực hiện trong một tuần để nhằm tránh sự phát triển của khối u, đặc biệt các khối u ác tính [6].

Kích thước ô lưới trong nghiên cứu được chọn từ $1 \mathrm{~mm}-2 \mathrm{~mm}$ tùy theo kích thước khối u. Theo Chung và cộng sự thì có sự khác biệt $2,3 \%$ về liêu chỉ định khi thay đổi kích thước ô lưới tính toán từ $2 \mathrm{~mm}$ xuống $1,5 \mathrm{~mm}$ [1].

Nghiên cứu của chúng tôi sử dụng bộ tiêu chí $3 \% / 2 \mathrm{~mm}, 2 \% / 2 \mathrm{~mm}, 2 \% / 1 \mathrm{~mm}$ với tỉ lệ số điểm có gamma <1 là trên $90 \%$ khi thực hiện kiểm định kế hoạch trước điều trị với thiết bị tại trung tâm. Điều này cũng phù hợp với một số một số nghiên cứu chỉ ra khi sử dụng thiết bị đo liều Arccheck thì tiêu chí $3 \% / 2 \mathrm{~mm}$ được sử dụng trong kiểm chuẩn kế hoạch xạ phẫu [4]. Khi sử dụng thiết bi Dolphin sử dụng các tiêu chí ( $\% / 2 \mathrm{~mm}$ hoặc $2 \% / 1 \mathrm{~mm})$. Ngoài ra, nghiên cứu của Dr. Yang Tuck Loong đã sử dung Matrixx (iBa) với tiêu chí $3 \% / 3 \mathrm{~mm}$ để kiểm định kế hoạch trước điều trị.

Ngoài ra trong nghiên cứu cũng đánh giá quy trình thực hành xạ phẫu trên phantom đâu người nhằm đánh giá cả quá trình điều trị. Trên phantom tiến hành lập kế hoạch theo tiêu chí đã đưa ra và các chỉ số CIPaddick là 0,87 thỏa mãn trong khoảng PCI $(0,75-0,9)$; theo nghiên cứu của Ruggero Ruggieri và cộng sự (2018) và GI là 3,87 thỏa mãn theo nghiên cứu của David $\mathrm{J}$. Eaton và cộng sự năm 2018 và 2017 [7]. Kết quả đánh giá kế hoạch trước điều trị với thiết bị Dolphin và Arccheck thỏa mãn theo hướng dẫn AAPM TG218 và một số nghiên cứu khác.

\section{KẾT LUÂN}

Nghiên cứu khẳng định khả năng thực hiện kĩ thuật xạ trị lập thể trên nền tảng máy gia tốc và các thiết bị đi kèm của cơ sở trước khi thực hiện điều trị trên bệnh nhân.

\section{TÀI LIÊU THAM KHẢO}

1. Moyed Miften, Arthur Olch, et al, Tolerance Limits and Methodologies for IMRT MeasurementBased Verification QA: Recommendations of AAPM Task Group No. 218, 2018.

2. Lotte Wilke, Nicolaus Andratschke, et al, Prescribing, recording, and reporting of stereotactic treatments with small photon beamsreport 91, Journal of the ICRU, 2017.

3. Roper J, Chanyavanich V, Betzel G, et al. Single-isocenter multiple-target SRS: risk of compromised coverage. Int J Radiat Oncol Biol Phys. 2015; 93:540-546.

4. Benedict SH, Yenice KM, Followill D, et al, (2012), Stereotactic body radiation therapy: the report of AAPM Task Group 101, Med Phys, 39(1):563.

5. Grant M. Clark MD, Richard A. Popple PhD, et al, Plan quality and treatment planning technique for single isocenter cranial radiosurgery with 
volumetric modulated arc therapy, Practical Radiation Oncology 2, 2012, 306-313.

6. Dianne Hartgerinka, Ans Swinnena, et al, LINAC based stereotactic radiosurgery for multiple brain metastases: guidance for clinical implementation, Acta Oncologica, 2019.
7. David J. Eaton, Jonathan Lee, Rushil Patel, et al, Stereotactic radiosurgery for benign brain tumours: Results of multi-centre benchmark planning studies, Practical Radiation Oncology, 2018. 